\title{
IUCrJ
}

Volume 7 (2020)

Supporting information for article:

Structure of the MICU1-MICU2 heterodimer provides insights into the gatekeeping threshold shift

Jongseo Park, Youngjin Lee, Taein Park, Jung Youn Kang, Sang A Mun, Minwoo Jin, Jihyeong Yang and Soo Hyun Eom 


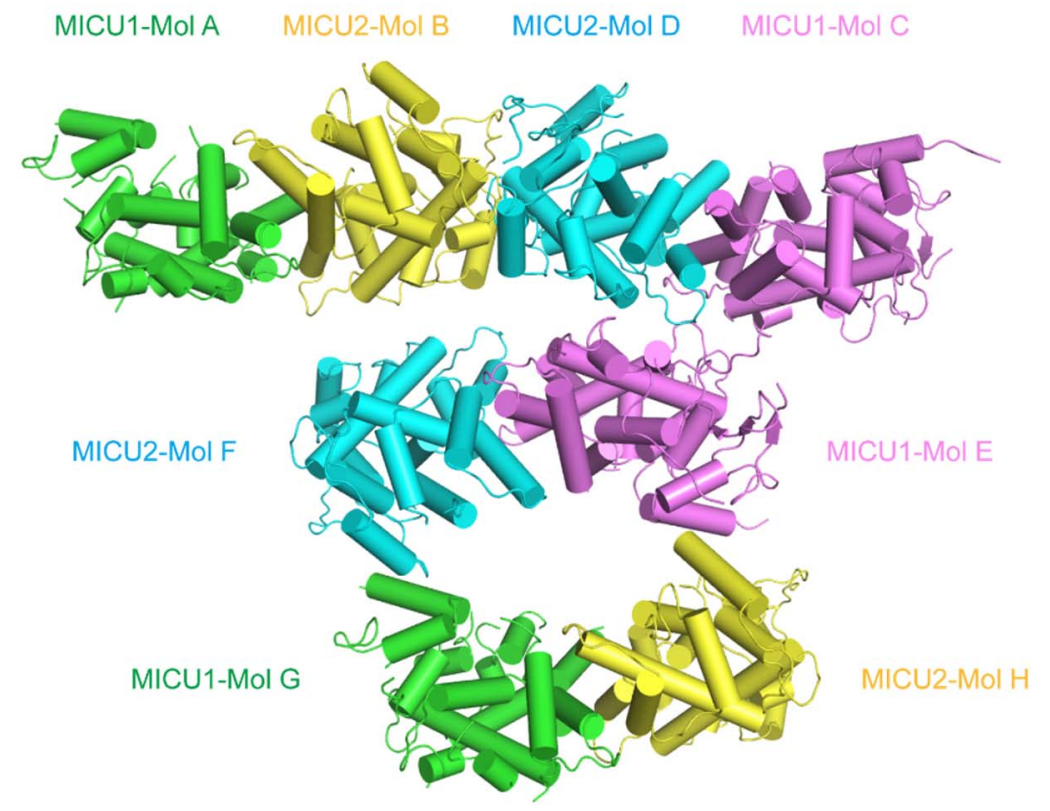

Figure S1 Four heterodimers consisting of an asymmetric unit. Overall view of the MICU1-MICU2 heterodimer structure occupying the ASU. There are four heterodimers which interact with each other through back-to-back interaction of MICU2. MICU1 is colored in green and violet. MICU2 is colored in yellow and cyan. 


\begin{tabular}{|c|c|c|c|c|c|}
\hline \multirow{2}{*}{ Interface area } & \multicolumn{3}{|c|}{ Face-to-Face (F-F) dimer } & \multicolumn{2}{c|}{ Back-to-Back (B-B) dimer } \\
\cline { 2 - 5 } & MICU1-2 & $\begin{array}{c}\text { MICU1 } \\
(4 \mathrm{NSC})\end{array}$ & $\begin{array}{c}\text { MICU2 } \\
(6 \mathrm{AGH})\end{array}$ & MICU2 & $\begin{array}{c}\text { MICU2 } \\
(6 \mathrm{AGH})\end{array}$ \\
\hline Interface 1 $(\AA)$ & 654 & 532 & 361 & 860 & 1260 \\
\hline Interface 2 $(\AA)$ & 346 & 596 & 406 & 860 \\
\hline
\end{tabular}

(a)

\begin{tabular}{|c|c|c|c|}
\hline $\begin{array}{c}\text { Predicted } \\
\text { Binding Energy } \\
\text { (F-F dimer) }\end{array}$ & MICU1-2 & $\begin{array}{c}\text { MICU1 } \\
\text { (4NSC) }\end{array}$ & $\begin{array}{c}\text { MICU2 } \\
\text { (6AGH) }\end{array}$ \\
\hline $\begin{array}{c}\text { Interface 1 } \\
\text { (kcal/mol) }\end{array}$ & -7.6 & -6.9 & -5.5 \\
\hline $\begin{array}{c}\text { Interface 2 } \\
\text { (kcal/mol) }\end{array}$ & -5.2 & -7.0 & -6.0 \\
\hline
\end{tabular}

(b)

Figure S2 Interface area and predicted binding energies of the MICU1-MICU2 heterodimer and other homodimers in MICUs. (a) The interface area of the MICU1-MICU2 heterodimer and F-F or BB MICU homodimers calculated using the PDBePISA web server (PDB ID: 4NSC and 6AGH). MICU1-MICU2 heterodimer (Mol CD) and MICU2 homodimer (Mol BD) in the ASU are abbreviated to MICU1-2 and MICU2, respectively. Only the total interface area is provided in the BB dimer. (b) Predicted binding energies ( $\Delta \mathrm{G}, \mathrm{kcal} / \mathrm{mol})$ calculated using the PRODIGY web server. Interface area and predicted binding energies of MICU1-MICU2 heterodimer (Mol AB, CD), MICU1 homodimer (Chain AB, CE, DF), and B-B MICU2 homodimers in the ASU (Mol BD, Mol FH in crystal lattice) were calculated for each average. 


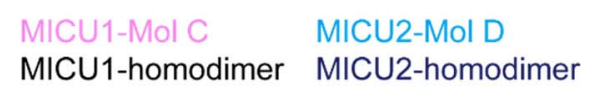

\section{MiCU1-homodimer MiCU2-homodimer}
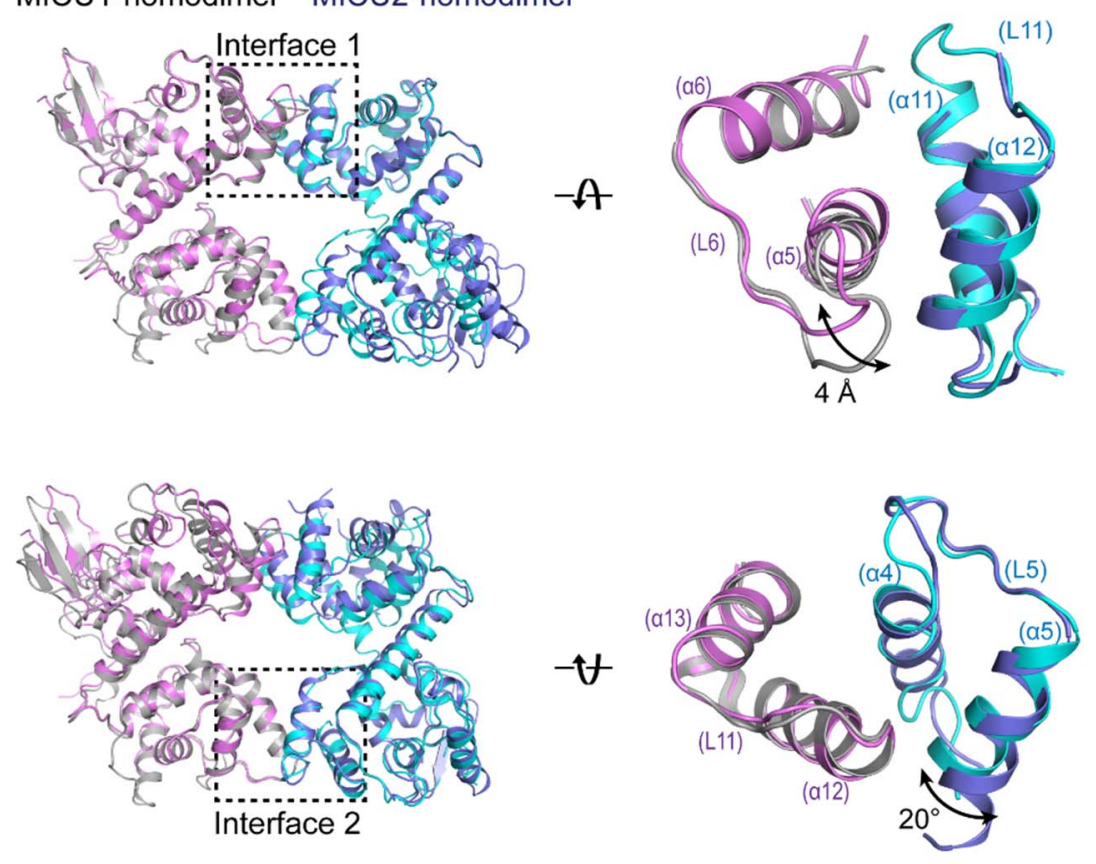

Figure S3 Conformational change of interface 1 and 2 compared to the apo state of MICU1 and MICU2. Cartoon representation of interface 1 and 2 of superimposed MICU1-MICU2 heterodimer and homodimers in MICU1 and MICU2 based on each interface. The conformational change of interloop (L6) of MICU1 EF-hand 1 and helix $\alpha 5$ of MICU2 EF-hand 3 is indicated with black arrow. 
MICU1

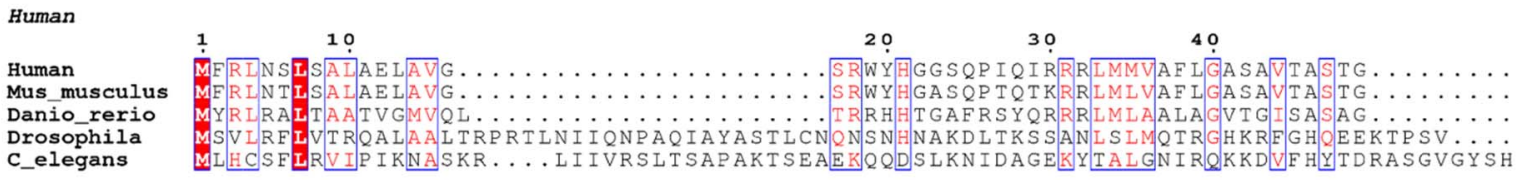

Human

Human

Mus_musculus

Danio_rerio

Drosophil

C_elegans

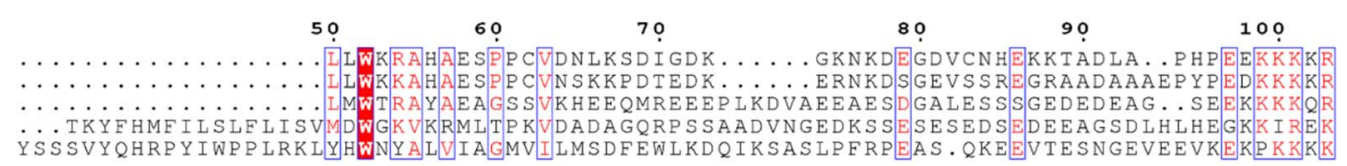

Human

Human

Mus_musculus

Danio_rerio

Drosophila

C_elegans
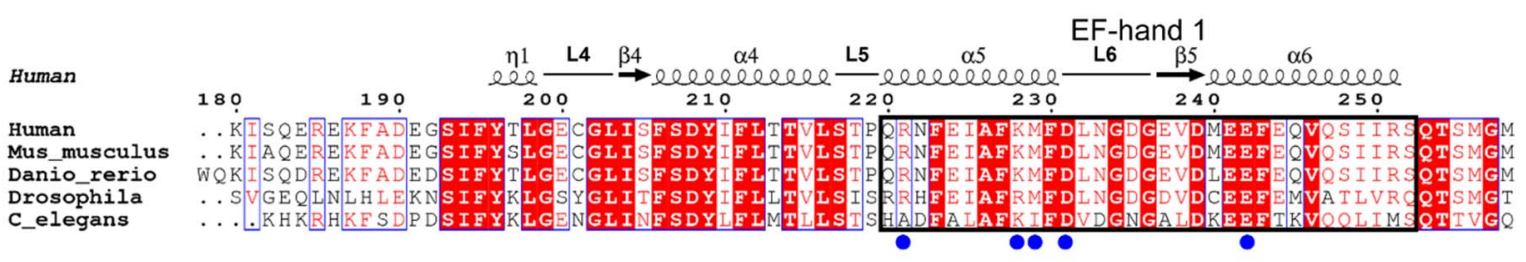

Human

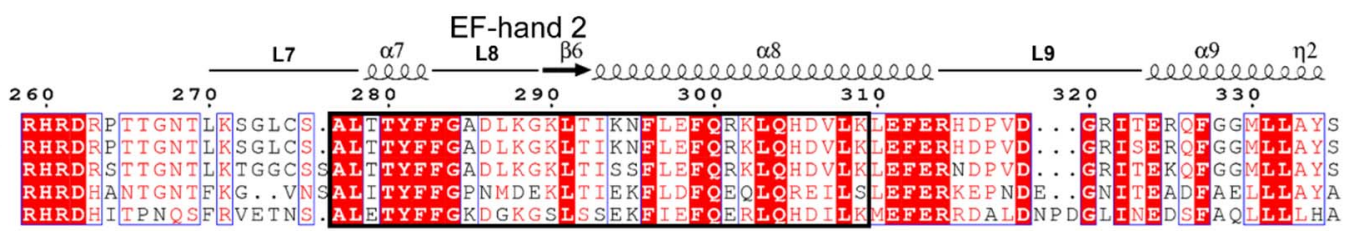

Human

Mus_musculus

Drosophila

C_elegans
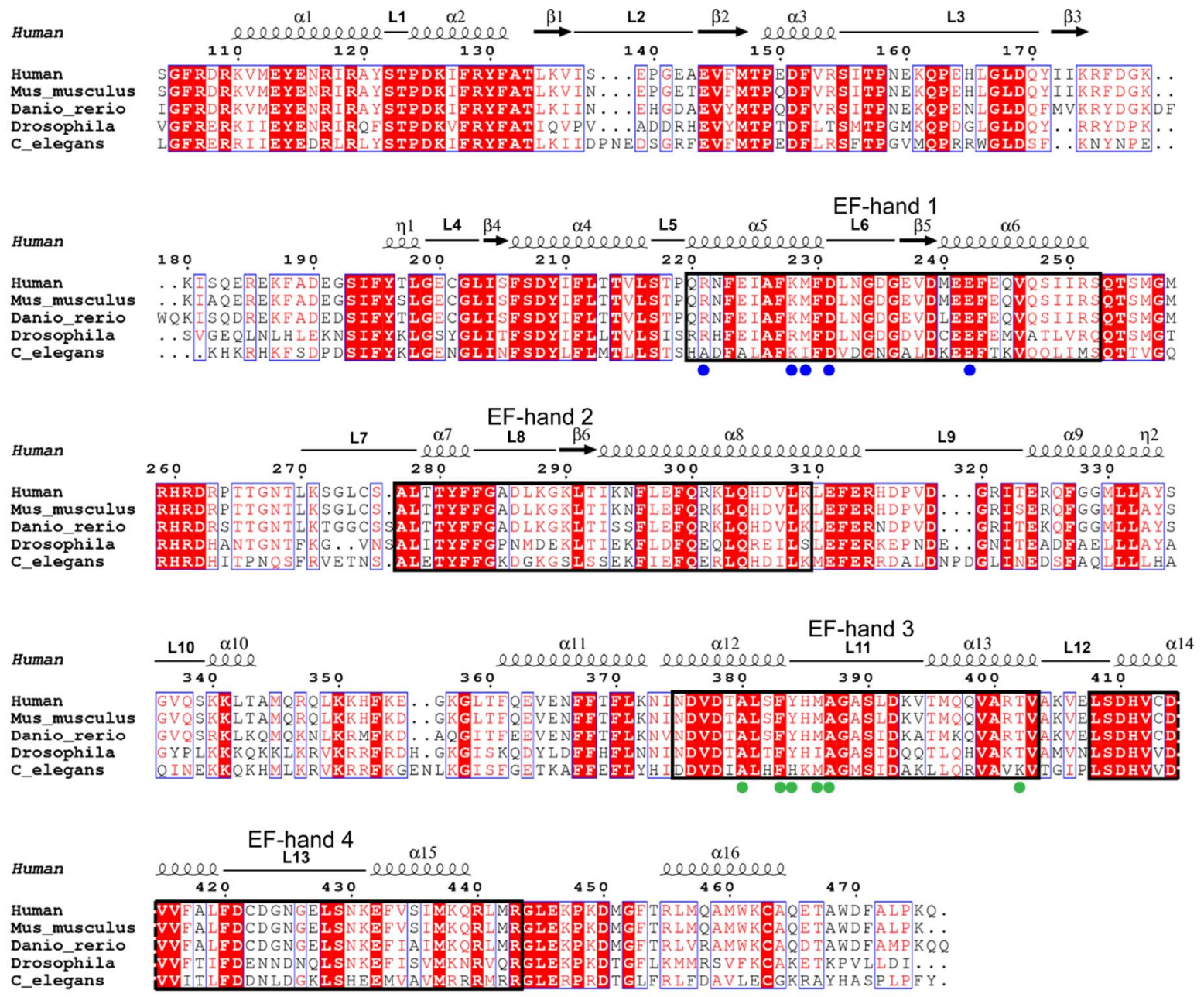

Figure S4 Multiple sequence alignment of MICU1. The amino acid sequence alignment of MICU1 sequences from various species. Key residues of interface 1 and 2 are marked by blue and green dots, respectively. All of the EF-hands are marked by black solid lined box. 
Human

Human

Mus_musculus

C_elegans

Danio_rerio Drosophila

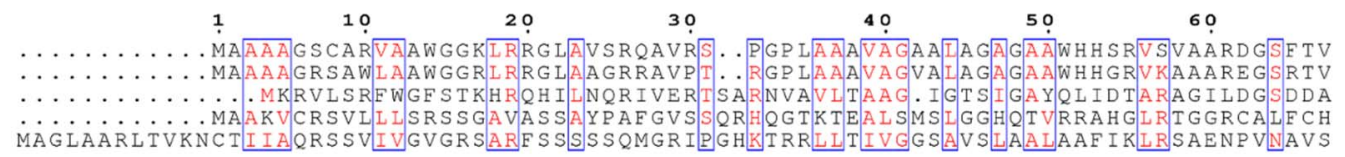

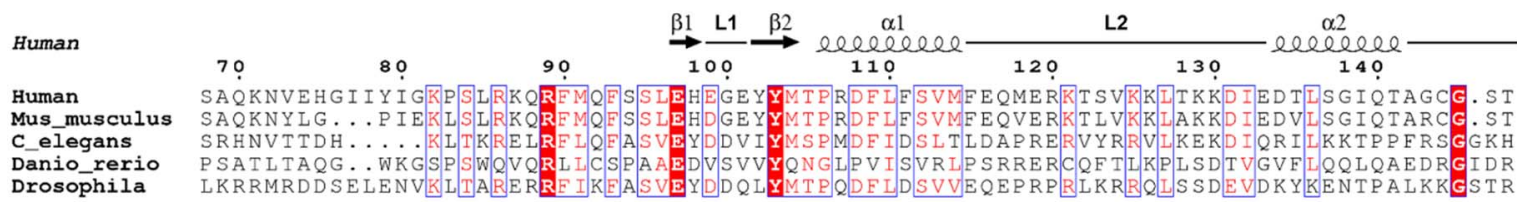

Human

Human

Mus_musculus

C_elegans

Danio rerio

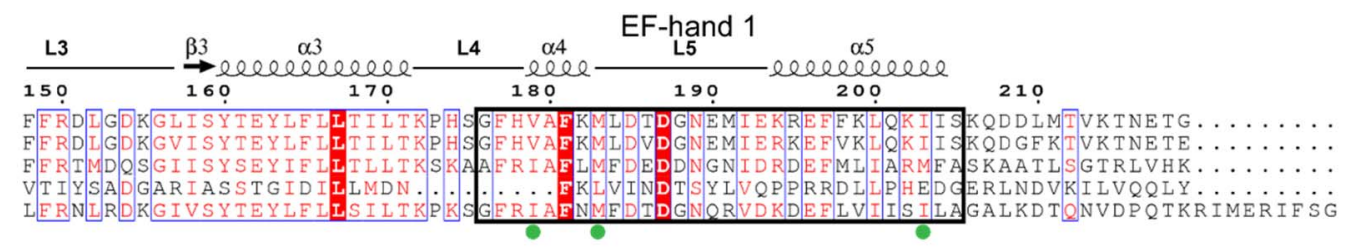

Human

Human

Mus_musculus

C_elegans

Danio rerio

Drosophila

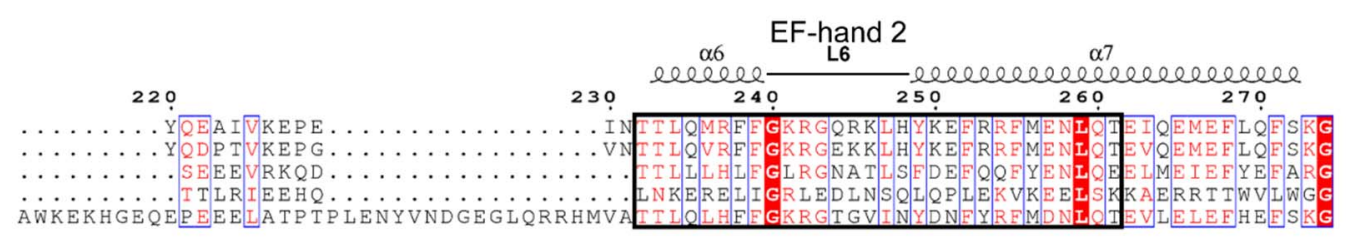

Human

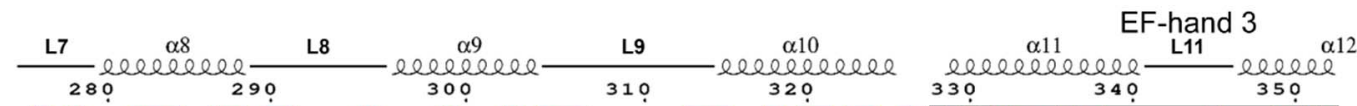

Human

Mus_musculus

C_elegans

Danio_rerio

Drosophila

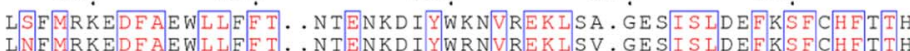

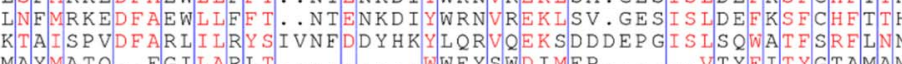

MAYMATQ. . FG I LAR T T. . . . ...WWEYSWD IMEP

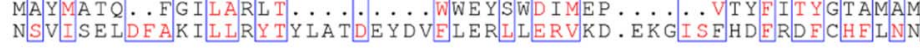
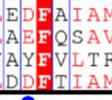

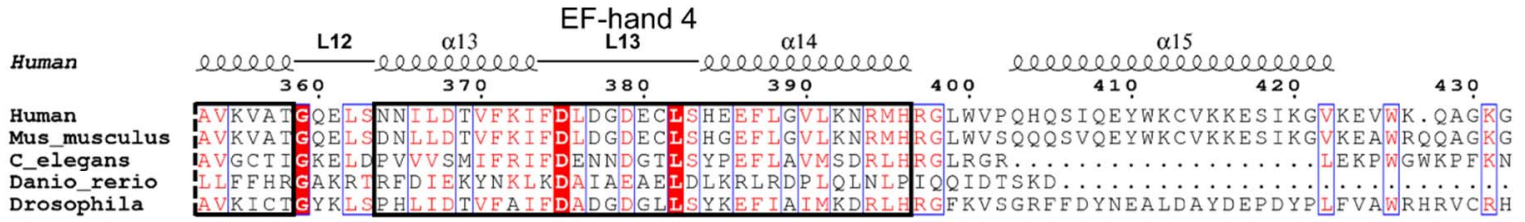

Human

Human

Mus musculus

C_elegans

Danio rerio

Drosophila

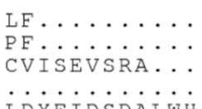

LDYEIDSDALWH

Figure S5 Multiple sequence alignment of MICU2. The amino acid sequence alignment of MICU2 sequences from various species. Key residues of interface 1 and 2 are marked by blue and green dots, respectively. All of the EF-hands are marked by black solid lined box. 


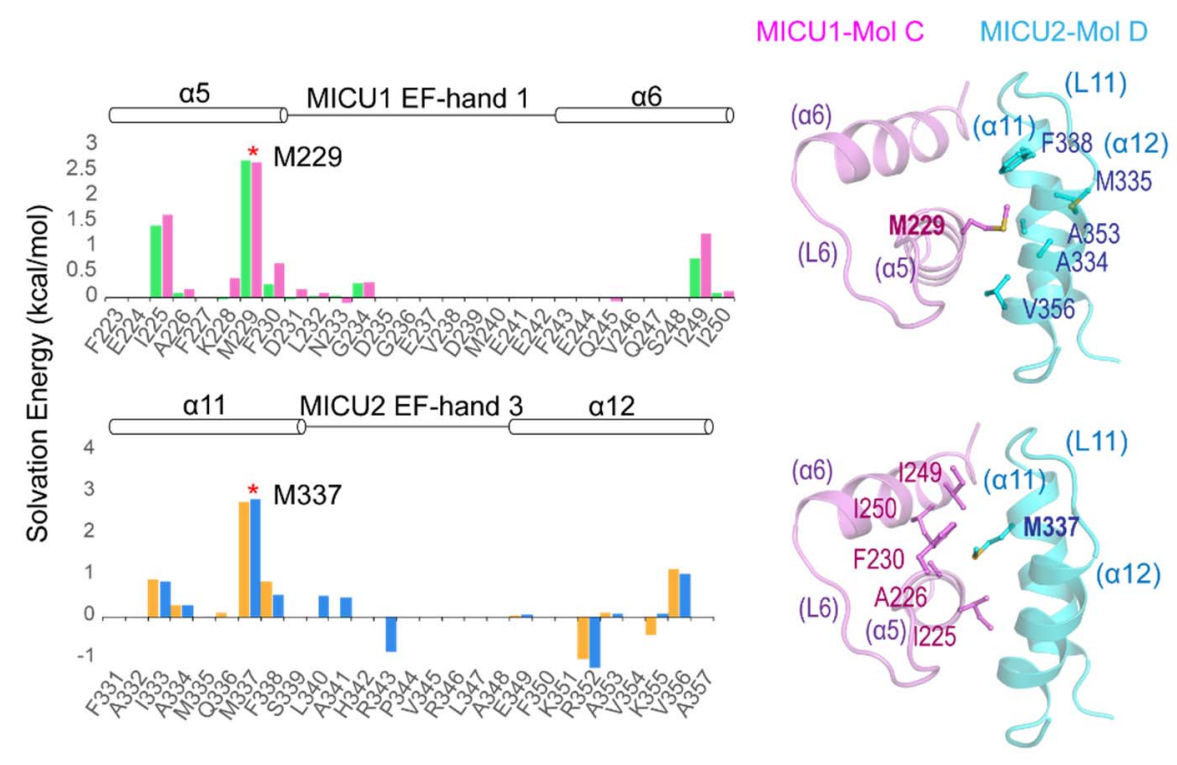

Figure S6 Solvation energies and cartoon representations of MICU1 and MICU2 in interface 1. Plots of solvation energy $(\mathrm{kcal} / \mathrm{mol})$ and cartoon representations for EF-hand 1 and EF hand 3 of MICU1 or MICU2 constituting interface 1. Cartoon representations show the several residues of interface 1 participating in hydrophobic interactions with two methionine knobs of MICU1-MICU2 heterodimer, Mol CD. Asterisks show the residues that have the highest solvation energy value in each EF-hand, M229(MICU1) and M337(MICU2). 

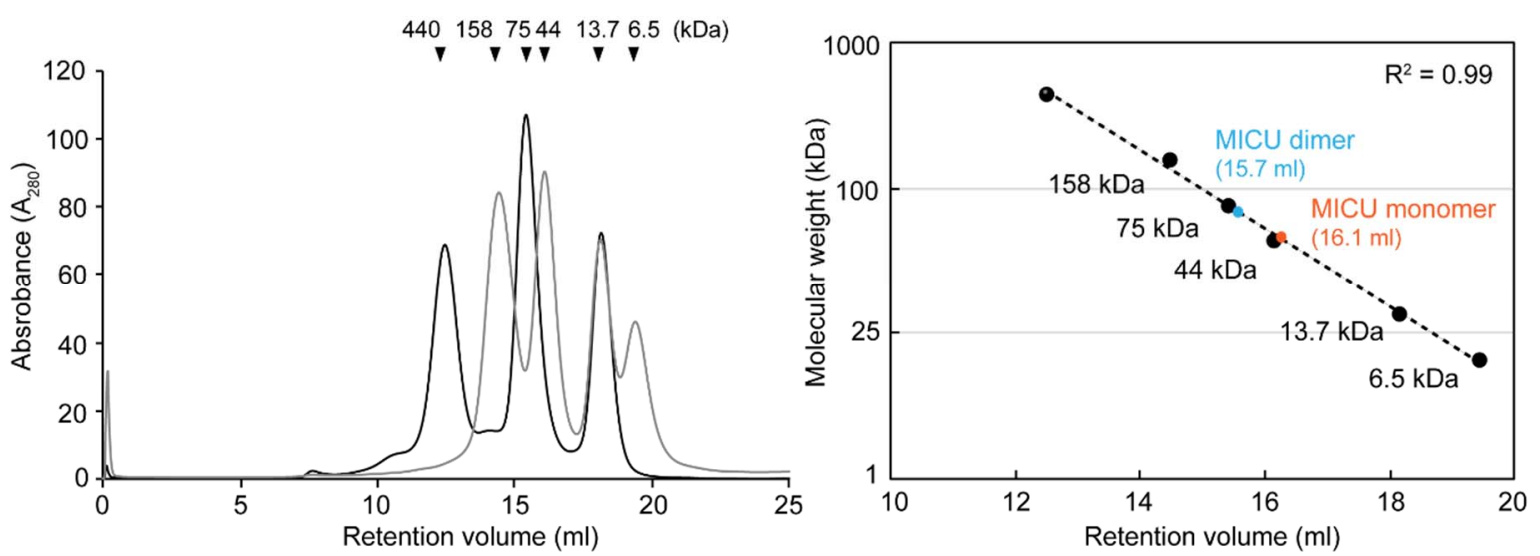

Figure S7 Calibration chromatogram and standard curve of size-exclusion chromatography (SEC).

(a) Size-exclusion chromatogram of molecular weight (m.w.) standards containing ferritin (440 kDa), aldolase (158 kDa), Conalbumin (75 kDa), Ovalbumin (43 kDa), ribonuclease A (13.8 kDa), and aprotinin $(6.5 \mathrm{kDa})$. (b) A standard curve based on the chromatogram. Cyan and orange dots indicate the eluted position of MICU dimer $(89.7 \mathrm{kDa})$ and MICU monomer (44.9 kDa), respectively. 